\title{
Interfacial hydrogen-bonding dynamics in surface-facilitated dehydrogenation of water on $\mathrm{TiO}_{2}(110)$
}

Shijing Tan ${ }^{\dagger, \ddagger}$, Hao Feng ${ }^{\dagger, \ddagger}$, Qijing Zheng ${ }^{\dagger}$, Xuefeng Cui ${ }^{\dagger}$, Jin Zhao ${ }^{\dagger}$, Yi Luo ${ }^{\dagger}$, Jinlong Yang ${ }^{\dagger}$, Bing Wang ${ }^{*, \dagger}$, J. G. Hou ${ }^{*, \dagger}$

$\dagger$ Hefei National Laboratory for Physical Sciences at the Microscale and Synergetic Innovation Center of Quantum Information \& Quantum Physics (CAS), University of Science and Technology of China, Hefei, Anhui 230026, China.

These authors contributed equally to this study.

*e-mail: bwang@ustc.edu.cn,jghou@ustc.edu.cn 

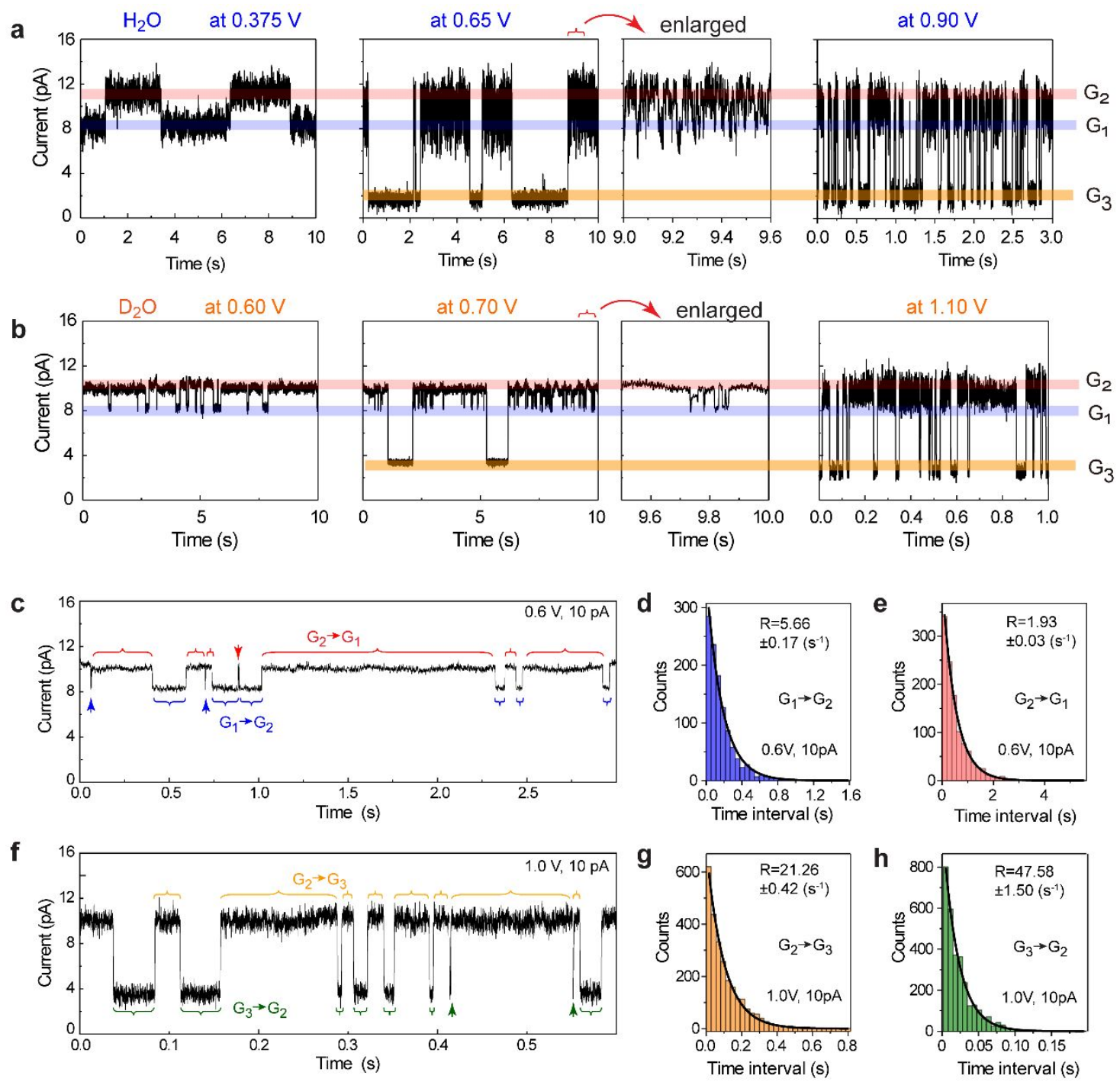

Figure S1. Conductance states and switching rates of $\mathrm{H}_{2} \mathrm{O}$ and $\mathrm{D}_{\mathbf{2}} \mathrm{O}$ measured in $\boldsymbol{I - t}$ curves. (a) $I-t$ curves obtained at $0.375,0.65$, and $0.90 \mathrm{~V}$ with the STM tip located over a $\mathrm{H}_{2} \mathrm{O}$ molecule. The voltage range of 9.0 9.6 $\mathrm{s}$ in the $I-t$ curve of $0.65 \mathrm{~V}$ is enlarged to show the switching between $\mathrm{G}_{1}$ and $\mathrm{G}_{2}$ of $\mathrm{H}_{2} \mathrm{O}$. (b) $I$ - $t$ curves obtained at $0.60,0.70$, and $1.10 \mathrm{~V}$ with the STM tip located over a $\mathrm{D}_{2} \mathrm{O}$ molecule. The voltage range of $9.5 \sim 10.0 \mathrm{~s}$ in the $I-t$ curve of $0.70 \mathrm{~V}$ is enlarged to show the switching between $\mathrm{G}_{1}$ and $\mathrm{G}_{2}$ of $\mathrm{D}_{2} \mathrm{O}$. The horizontal broad lines in blue, red and orange indicate the conductance states of $\mathrm{G}_{1}, \mathrm{G}_{2}$ and $\mathrm{G}_{3}$ of $\mathrm{H}_{2} \mathrm{O}\left(\mathrm{D}_{2} \mathrm{O}\right)$ in different stationary geometries. The setpoint current is $10 \mathrm{pA}$ performed at $80 \mathrm{~K}$. (c) A typical $I-t$ curve of $\mathrm{D}_{2} \mathrm{O}$ obtained at $0.6 \mathrm{~V}$ and $10 \mathrm{pA}$. (d,e) Distributions of the time intervals for the stationary geometries of $\mathrm{G}_{1}$ and $\mathrm{G}_{2}$, respectively. (f) A typical I- $t$ curve of $\mathrm{D}_{2} \mathrm{O}$ obtained at $1.0 \mathrm{~V}$ and $10 \mathrm{pA}$. (g,h) Distributions of the time intervals for the stationary geometries of $\mathrm{G}_{2}$ and $\mathrm{G}_{3}$, respectively. The distribution is exponentially fitted (Supplementary Ref. 1) to obtain the switching rate, $R$. The data for exponential fitting were obtained from the measurements of 50 100 runs under every condition. The different time intervals of the states are marked by the brackets (arrows for the very short intervals) in (c) and (f). 

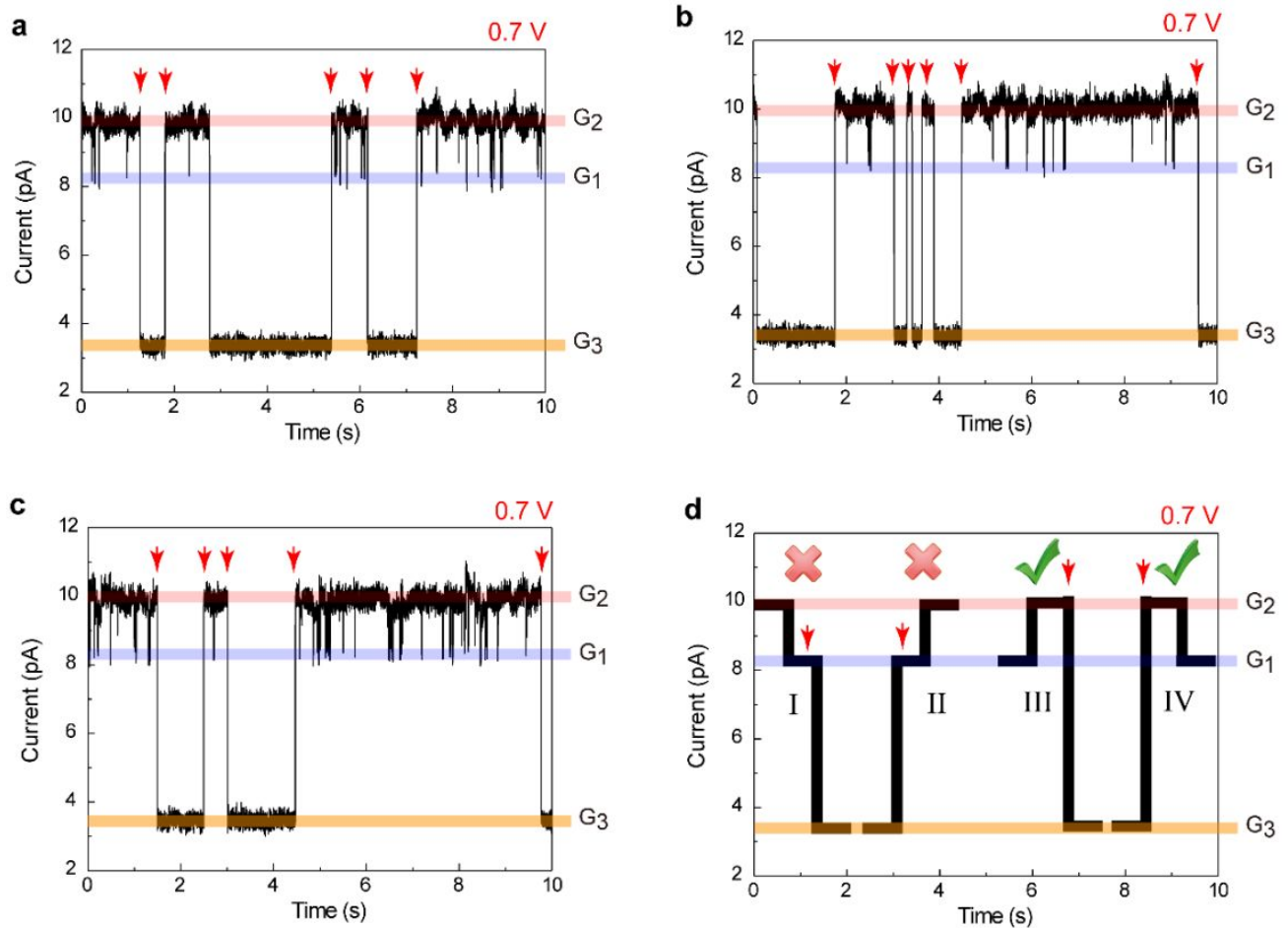

Figure S2. Switching between $\mathbf{G}_{\mathbf{1}}$ and $\mathbf{G}_{\mathbf{2}}$ and between $\mathbf{G}_{\mathbf{2}}$ and $\mathbf{G}_{\mathbf{3}}$. (a-c) Three representative $I-t$ curves obtained for a $\mathrm{D}_{2} \mathrm{O}$ molecule at $0.7 \mathrm{~V}$. The red arrows indicate the switching between $\mathrm{G}_{2}$ and $\mathrm{G}_{3}$. (d) A schematic drawing showing the direct switching between $\mathrm{G}_{1}$ and $\mathrm{G}_{3}$ (labeled by I and II), which is never observed in experiments.

Figure S2a-c shows some typical $I-t$ curves measured at moderate bias voltages. Figure S2d schematically summarizes our observations. There is not any event observable for the cases of I and II, and only the cases of III and IV are observed. No event for cases I and II strongly suggests that the switching to $\mathrm{G}_{3}$ always occurs from $\mathrm{G}_{2}$. 

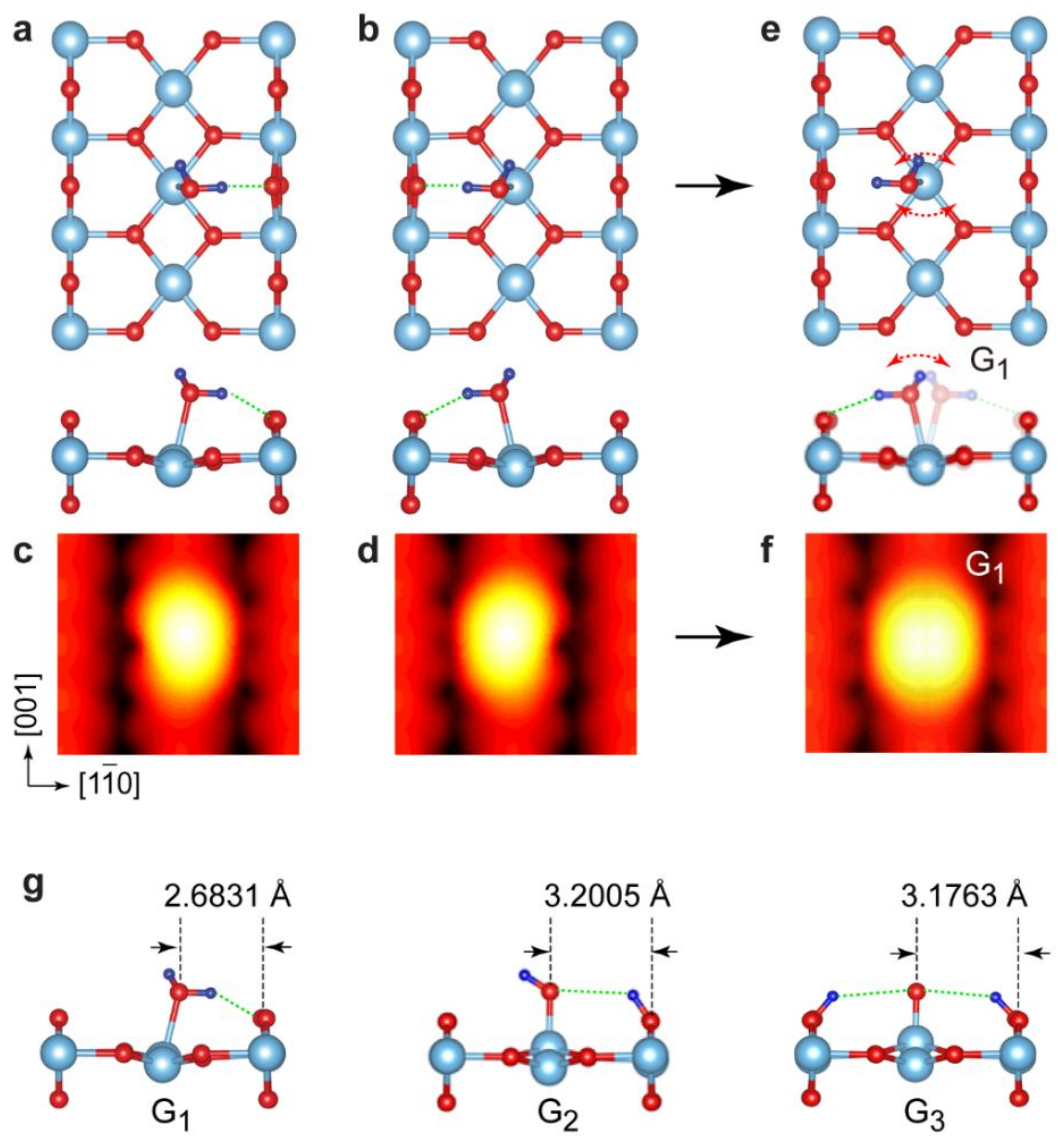

Figure S3. Simulated images of a $\mathrm{H}_{2} \mathrm{O}$ entity by averaging over the fast interconversion motions. (a,b) Structural models of the $\mathrm{G}_{1}$ geometry with the $\mathrm{H}_{2} \mathrm{O}$ entity inclined to the right side and left side, respectively; upper panel: top view; lower panel: side view. (c,d) Simulated image corresponding to the structures in (a) and (b), respectively. (e) Structural model of the $\mathrm{G}_{1}$ geometry by considering the fast interconversion motions between two configurations; upper panel: top view; lower panel: side view. (f) Simulated image by averaging over the two configurations in (c) and (d). (g) The O-O distances in the geometries of $\mathrm{G}_{1}, \mathrm{G}_{2}$ and $\mathrm{G}_{3}$. 

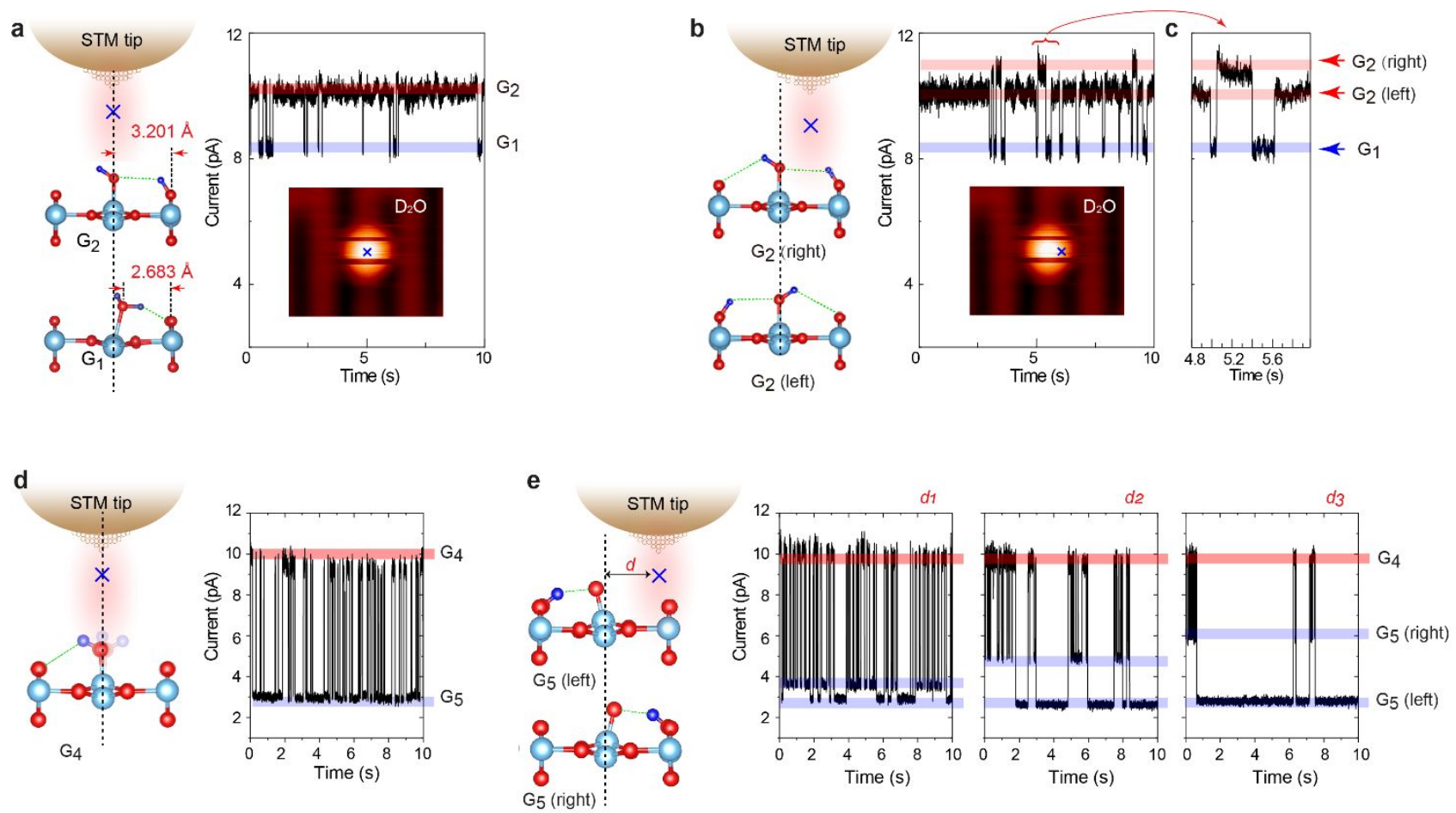

Figure S4. Dependence of the I-t curves on the lateral tip positions. (a) Schematic drawing and the $I-t$ curve recorded by positioning the tip centered over the $\mathrm{D}_{2} \mathrm{O}$. (b) Schematic drawing and the recorded $I$ - $t$ curve by positioning the tip off the center of $\mathrm{D}_{2} \mathrm{O}$, and (c) enlarged segment of the $I-t$ curve from (c). The inset images in (a) and (b) were recorded at $0.7 \mathrm{~V}$ and $10 \mathrm{pA}$, and the $I-t$ curves were recorded at $0.6 \mathrm{~V}$, with the initial setpoint of $10 \mathrm{pA}$. The cross shows the tip position. (d) Schematic drawing and recorded $I-t$ curve by positioning the tip centered over an $\mathrm{OH}_{\mathrm{t}}$. (e) Schematic drawing and recorded $I$ - $t$ curve by positioning the tip with different distances, $d$, off the center of $\mathrm{OH}_{\mathrm{t}}$. The three $I$ $t$ curves were recorded at $1.1 \mathrm{~V}$, with $d_{1}<d_{2}<d_{3}$. 


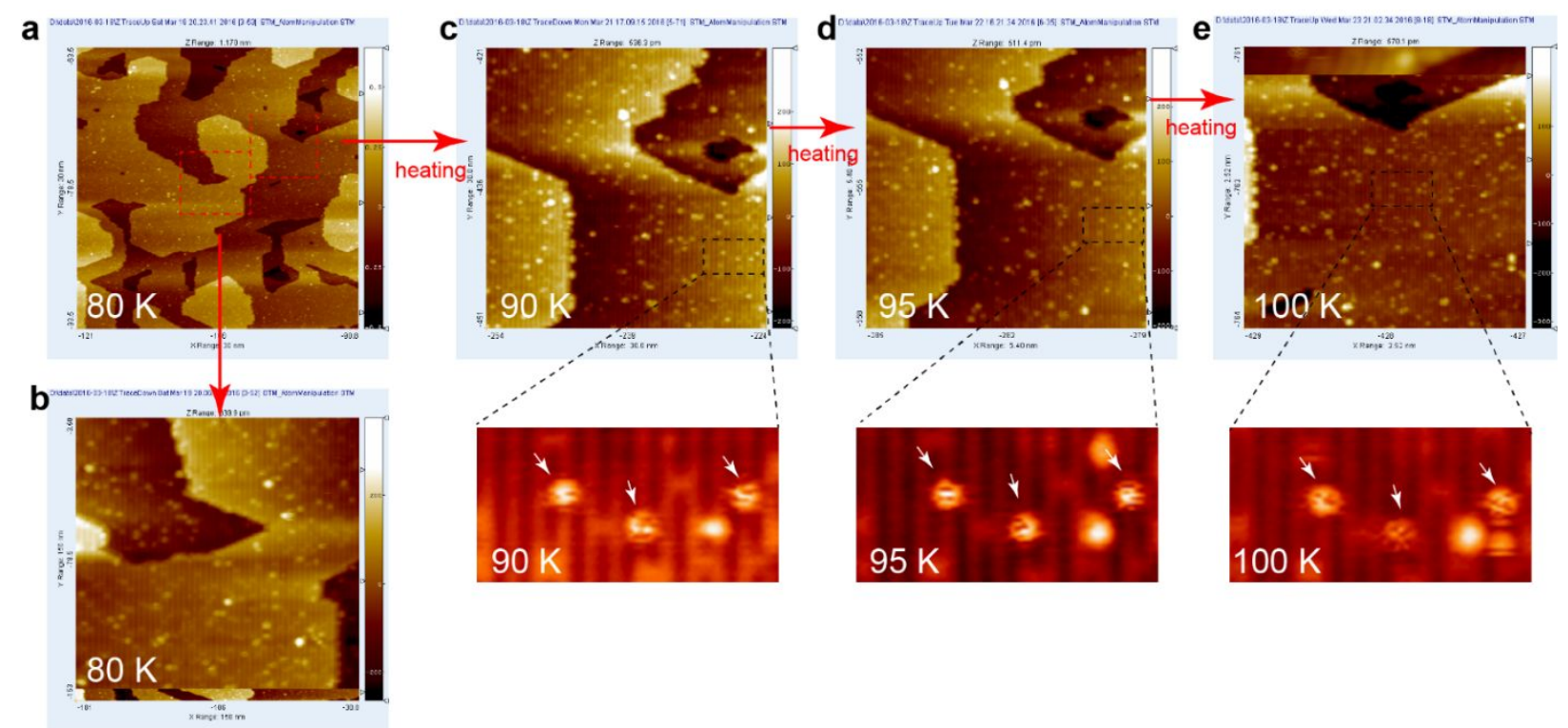

Figure S5. Tracking the same area from 80 to 100 K. (a) Large-scale STM image of $100 \times 100 \mathrm{~nm}^{2}$ obtained at $80 \mathrm{~K}$. (b) STM image obtained in the area marked by the lower-left dashed rectangle in (a). (c-d) STM images within the same area $\left(30 \times 30 \mathrm{~nm}^{2}\right)$ obtained at 90,95 , and $100 \mathrm{~K}$ by gradually heating the sample and repeatedly checking the drift that allowed us to track the same area.

To obtain the temperature dependent switching rate in Figure $3 \mathrm{~d}$ in the main text, we increased the sample temperature very slowly by $1 \mathrm{~K}$ and kept the sample at a target temperature for $\sim 5$ hours to obtain a stable image. We successfully tracked the same area at elevated temperatures of 90,95 and $100 \mathrm{~K}$ in $\sim 5$ days, as shown in (a-e). The data points at 90, 95 and $100 \mathrm{~K}$ in Figure $3 \mathrm{~d}$ in the main text were obtained at the same molecule, and the one at $80 \mathrm{~K}$ was averaged from 3 different molecules, which give the fitted thermal activation barriers according to the Arrhenius equation. 


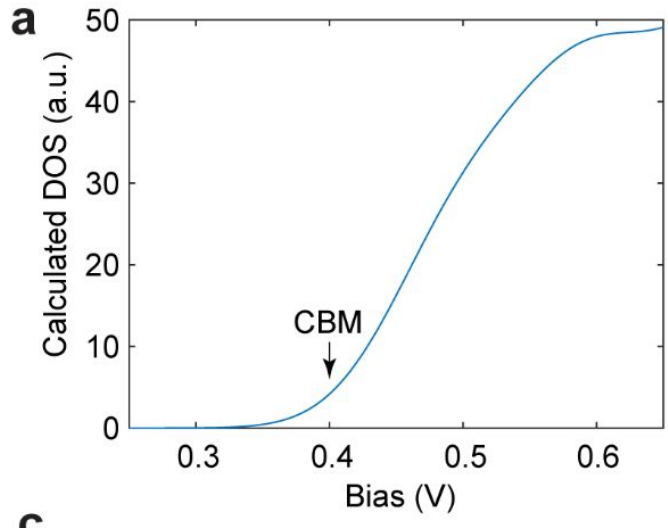

C

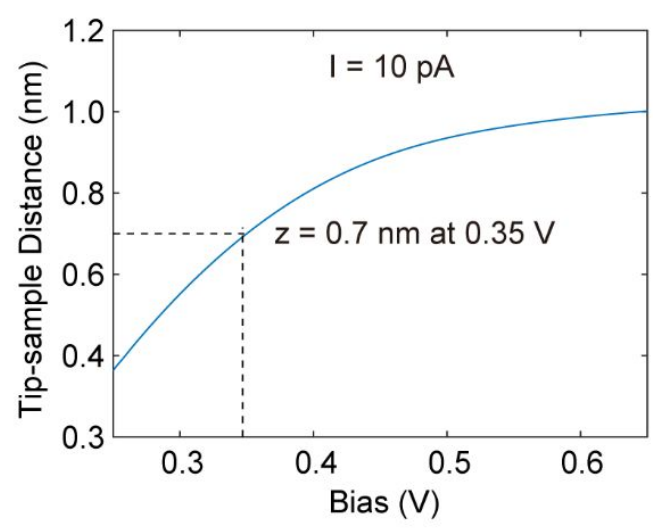

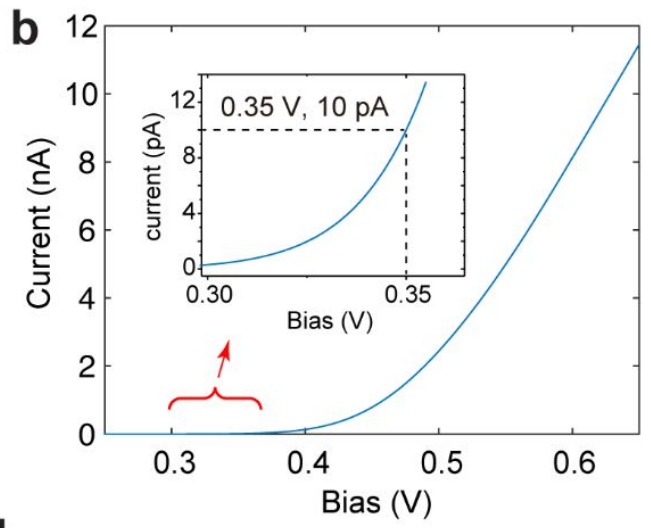

d

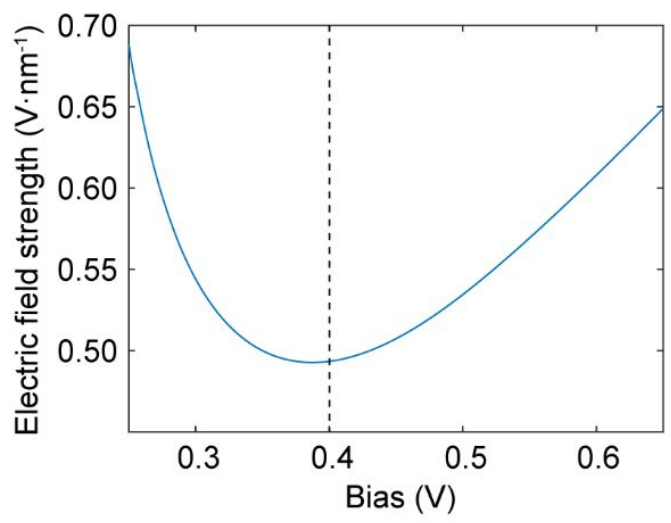

Figure S6. Simulation of the dependence of electric field on the bias voltage. (a) Calculated DOSs near the $\mathrm{CBM}$ of $\mathrm{TiO}_{2}(110)$, as adopted from the total DOS in Figure $3 \mathrm{f}$ in the main text. (b) Calculated current as a function of the bias voltage at a constant height of $z=7 \AA$. The inset shows the enlarged range at $V_{b}<0.36 \mathrm{~V}$. (c) Calculated tip-sample distance as a function of the bias voltage at a constant current of $I=10 \mathrm{pA}$. (d) Calculated electric field strength as a function of the bias voltage at a constant current of $10 \mathrm{pA}$ (note that at $0.35 \mathrm{~V}$ and $10 \mathrm{pA}$, we have $z \sim 7 \AA$, which is obtained from our measurements as shown in Figure S8 below).

Using our calculated DOSs (Figure S6a, adopted from the Figure $3 \mathrm{f}$ in the main text), where the conduction band edge is at $0.4 \mathrm{eV}$ above the $E_{\mathrm{F}}$ (Ref. 47 in the main text), we calculated the possible effect of the DOSs of $\mathrm{TiO}_{2}(110)$ on the electric field.

Using the relation $I \propto \int_{0}^{V} \rho_{\mathrm{s}}(V) \exp \left(-\frac{2 \sqrt{2 m \phi}}{\mathrm{h}} z\right) d V$, where $\rho_{\mathrm{s}}(V)$ is the calculated total DOSs of $\mathrm{H}_{2} \mathrm{O} / \mathrm{TiO}_{2}(110), m$ is the mass of an electron, $\phi \quad(=5.2 \mathrm{eV}$, obtained by measuring the cutoff energy using UPS using the $\mathrm{TiO}_{2}$ sample in our experiment) is the work function of $\mathrm{TiO}_{2}, z$ is the tip-sample distance, and $\mathrm{h}$ is the reduced Plank constant, we obtain the simulated $I-V, Z-V$, and electric field dependence on $\mathrm{V}(E-V)$ relations, as shown in Figure S6b,c,d. This figure shows an increasing electric field with decreasing $V_{\mathrm{b}}$ from $0.4 \mathrm{~V}$, which highly supports our suggestion of the involvement of the electric field. 
a

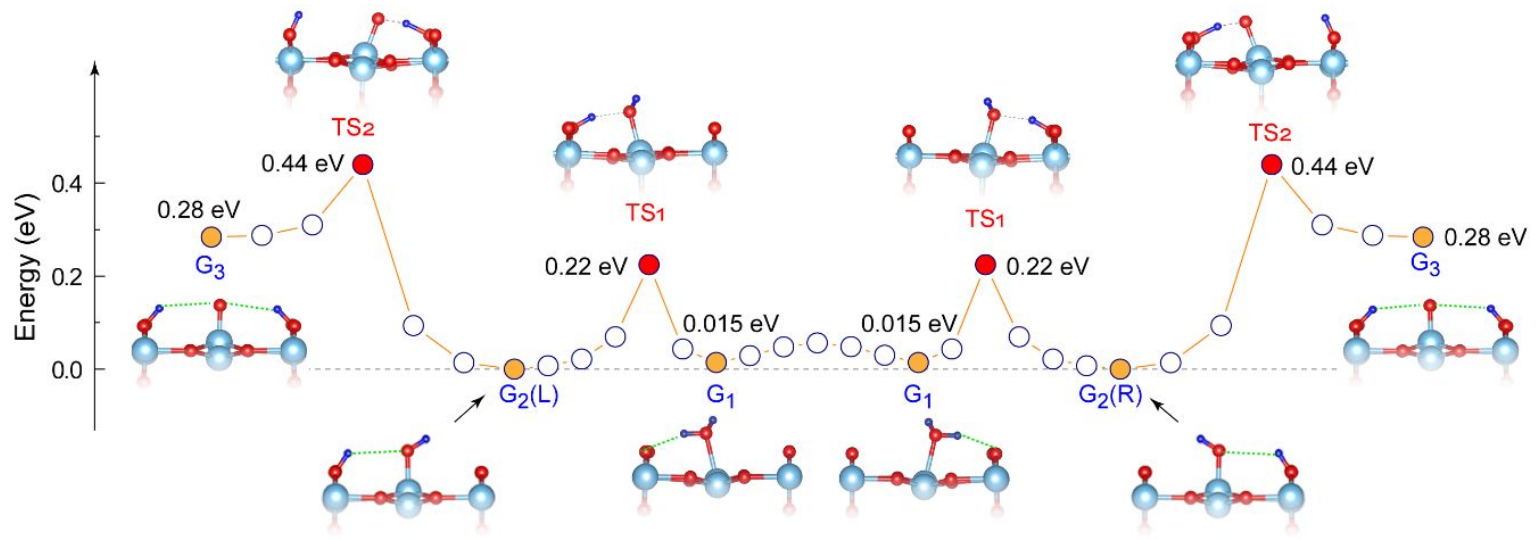

b

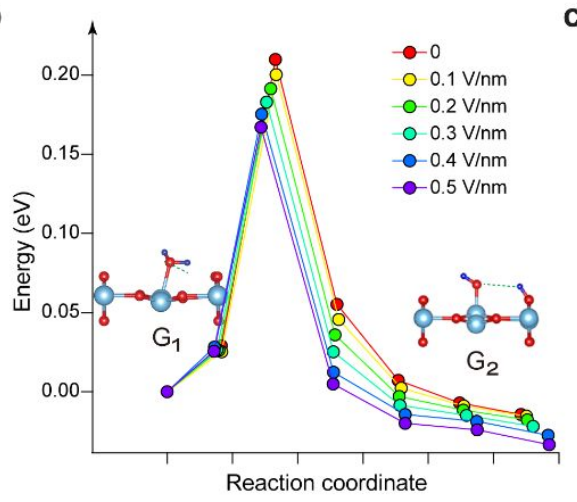

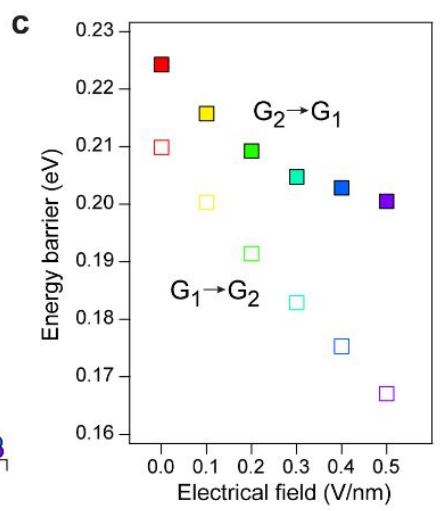

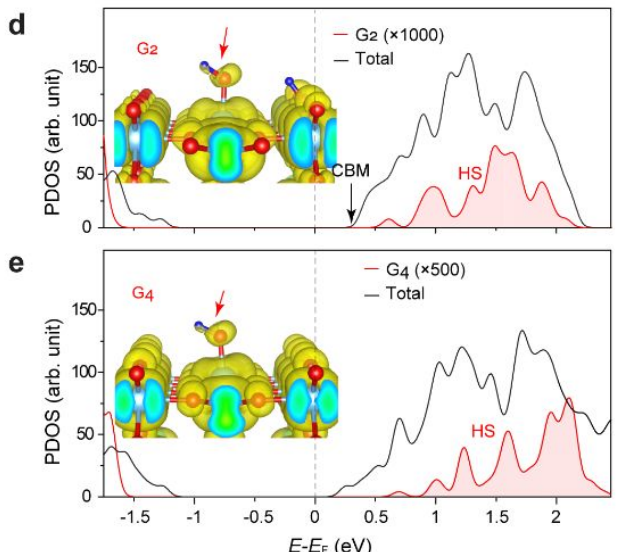

Figure S7. Calculations of the electric-field-induced barrier change and comparison of the hybridization states at different geometries. (a) Calculated ground state potential energy surface (PES) for the two-step dehydrogenation process of $\mathrm{H}_{2} \mathrm{O}$. (b) The PES for the switching between the stationary geometries $\mathrm{G}_{1}$ and $\mathrm{G}_{2}$, with an increasing external electrical field from 0 to $0.5 \mathrm{~V} / \mathrm{nm}$. The insets show the $G_{1}$ and $G_{2}$ geometries. (c) Plot of the energy barriers for the switching from $G_{1}$ to $G_{2}$ and from $G_{2}$ to $G_{1}$ as a function of the electrical field strength. (d) Calculated partial and total DOS of the geometry of $\mathrm{G}_{2}$ for $\mathrm{H}_{2} \mathrm{O}$. The inset shows the spatial distribution of hybridization states (HSs), indicating the weak antibonding orbitals located at the $\mathrm{O}-\mathrm{H}$ bond of the dissociative $\mathrm{H}_{2} \mathrm{O}$. (e) Calculated partial and total DOS of the geometry of $\mathrm{G}_{4}$ for $\mathrm{OH}_{\mathrm{t}}$. The inset shows the spatial distribution of the HSs in $\mathrm{OH}_{\mathrm{t}}$. The red shadows show the energy range of the HSs in (d) and (e). The similar weak antibonding orbitals locating at the $\mathrm{O}-\mathrm{H}$ bond for geometries of $\mathrm{G}_{2}$ and $\mathrm{G}_{4}$ are responsible for the electronic excitation of $H$ transfer from $G_{2}$ to $G_{3}$ and from $G_{4}$ to $G_{5}$, respectively. The CBM denotes the conduction band minimum, and the Fermi level $\left(E_{F}\right)$ is set at $0.4 \mathrm{eV}$ below the CBM. 


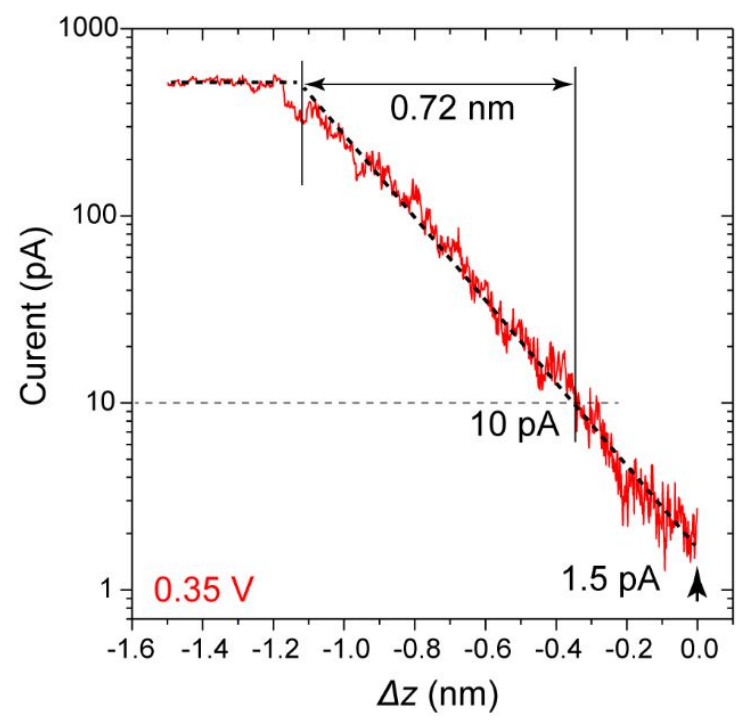

Figure S8. Measurement of tip-sample distance at $0.35 \mathrm{~V}$ and $10 \mathrm{pA}$. The $I-z$ curve obtained at 0.35 $\mathrm{V}$ with the feedback loop off. The initial setpoint current is $1.5 \mathrm{pA}$, corresponding to $\Delta z=0$. The plateau indicates the contact between the tip and the sample (Supplementary Ref. 2). 

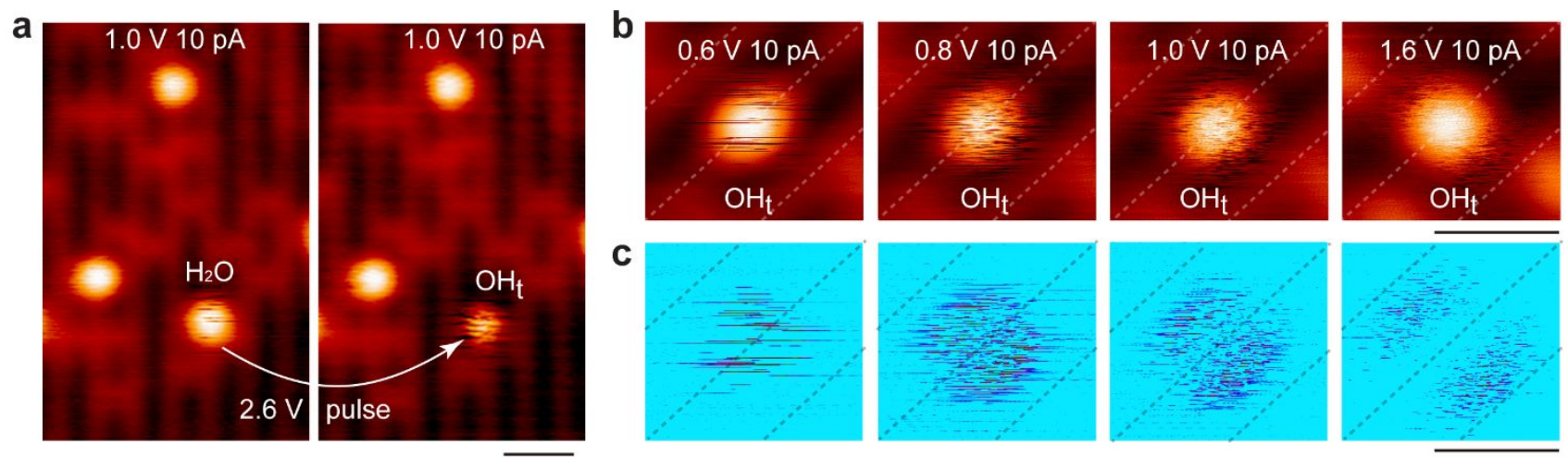

Figure S9. Fuzzy features in the images of $\mathbf{O H}_{\mathbf{t}}$ (a) STM images showing the preparation of an $\mathrm{OH}_{t}$ by removing one $\mathrm{H}$ atom through applying a voltage pulse of $2.6 \mathrm{~V}$. Scale bar: $1 \mathrm{~nm}$. (b,c) A set of STM images (upper) and corresponding high-pass filtered images (lower) of $\mathrm{OH}_{t}$ acquired under different conditions.

\section{Supplementary References:}

1. Pan, S.; Fu, Q.; Huang, T.; Zhao, A. D.; Wang, B.; Luo, Y.; Yang, J. L.; Hou, J. G., Design and control of electron transport properties of single molecules. P. Natl. Acad. Sci. USA 2009, 106, 15259-15263.

2. Cui, X.F.; Wang, Z.; Tan, S. J.; Wang, B.; Yang, J. L.; Hou, J. G. Identifying Hydroxyls on the $\mathrm{TiO}_{2}$ (110)-1×1 Surface with Scanning Tunneling Microscopy. J. Phys. Chem. C 2009, 113, 1320413208 